\title{
First Principle Calculation of Electronic and Optical Properties of Rare Gas Solids Kr and Ar
}

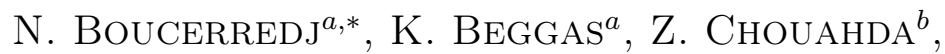 \\ S. GHEMID ${ }^{b}$ AND H. MERADJI ${ }^{b}$ \\ ${ }^{a}$ Semiconductor Laboratory, Physics Department \\ Faculty of Sciences, Badji Mokhtar Annaba University, B.O. 12, 23000 Annaba, Algeria \\ ${ }^{b}$ Radiations Physics Laboratory, Physics Department \\ Faculty of Sciences, Badji Mokhtar Annaba University, B.O. 12, 23000 Annaba, Algeria
}

Received: 02.02.2020 \& Accepted: 10.06.2020

Doi: 10.12693/APhysPolA.138.428

*e-mail: boucerredj@yahoo.fr

\begin{abstract}
In this study, we investigate the electronic and optical properties of rare gas solids $\mathrm{Kr}$ and $\mathrm{Ar}$ in detail using the first principle calculation. A full potential linearized augmented plane wave method has been employed in conjunction with the Tran Blaha modified Becke-Johnson potential plus local density approximation and the generalized gradient approximation exchange correlation potentials. We have found that the studied rare gas solids have insulator nature with large gap energy of order of $10.523 \mathrm{eV}$ and $13.673 \mathrm{eV}$ for $\mathrm{Kr}$ and $\mathrm{Ar}$, respectively. The obtained electronic results are in good agreement with the available experimental and theoretical data. The optical properties, including the reflectivity $R(w)$, the absorption coefficient $\alpha(w)$, the energy loss function $L(w)$, the refractive index $n(w)$ and the extinction coefficient $k(w)$ are deduced from real and imaginary parts of the calculated dielectric function within the modified Becke-Johnson plus local density approximation and generalized gradient approximation exchange correlation potentials. We have found a direct band gap for the two rare gas solids, good refractive index, large absorption band between 10 and $20 \mathrm{eV}$. This is the first quantitative theoretical prediction of the optical properties for the two rare gas solids that requires experimental confirmation. The obtained results can be exploited for the application in optoelectronics.
\end{abstract}

topics: DFT, rare gas solids, optical properties, electronic properties

\section{Introduction}

In the last few years, a great attention of scientific researchers has been attracted to investigate theoretically and experimentally the physical properties of atomic clusters and their industrial applications in optoelectronics, lithography, medicine, lasers, chemical inert atmosphere, used also to make excimer lasers, when doping with fluorine, used in microlithography and micro fabrication of integrated circuits, argon lasers, laser pumping, and laser surgery. The rare gas clusters are also used as a source of energetic particles when they are irradiated by intense femtosecond lasers $[1,2]$.

Theoretical studies have become fundamental in the development of new materials and new devices for industrial applications. Several groups have investigated experimentally and theoretically optical spectra and electronic properties of rare gas solids, employing a variety of approximations [3-5]. Their importance lies in the simplicity of their crystal structure. Their energy gap are underestimated by local density approximation (LDA) and overestimated with generalized gradient approximation (GGA) in comparison with the experimental values.
The electronic structure calculations in solids are done using the Kohn-Sham equations with the local density approximation LDA or GGA for the exchange correlation energy and potential. The main reason is that these approximations yield to an accurate result which helps to interpret experimental results [6-10]. However, modified Becke-Johnson (mBJ) method is employed to correct the band gap and evaluate the density of state [11]. This parameterizations yields band gap in good agreement with experiments. The rare gas solids $\mathrm{Kr}$ and $\mathrm{Ar}$ have a large energy gap and a face centred cubic (fcc) structure. For their importance in industrial applications, we study the electronic and optical properties of these rare gas solids to clarify their physical properties.

In this paper, we use the Tran-Blaha modified Becke-Johnson (TB-mBJ) exchange potential $[6,12]$ with LDA and GGA exchange correlation potentials to investigate the electronic and optical properties of rare gas solids $\mathrm{Kr}$ and Ar using the full potential linearized augmented plane wave method (FP-LAPW) [13] implemented in Wien2k code $[14,15]$. The paper is organized as follows: The calculation method and the computational 


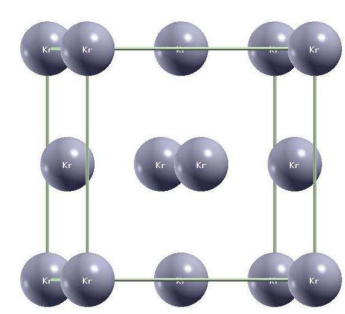

(a)

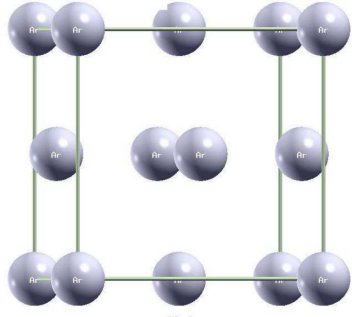

(b)
Fig. 1. Crystal structures of rare gas solids: (a) Kr and (b) Ar.

details are briefly described in Sect. 2. The results and discussion of electronic and optical properties and the comparison with available experimental and theoretical results are presented in Sect. 3. Finally, conclusion is given in Sect. 4 .

\section{Computational method}

In the present calculation, the electronic structure and optical properties of rare gas solids $\mathrm{Kr}$ and $\mathrm{Ar}$ are studied. The exchange correlation contribution is described within the TB-mBJ exchange potential plus LDA and GGA(96) based on the Perdew-Burke-Ernzerhof (GGA-PBE) [10] exchange correlation potentials. The energy separation between the valence and core states is $-7.0 \mathrm{Ry}$. The valence wave function inside the muffin-tin spheres are expanded in terms of spherical harmonics to $l=10$. We set the $R_{m t} K_{\max }$ to 8 , where $R_{m t}$ is the smallest atomic muffin-tin sphere radius and $K_{\max }$ is the magnitude of the largest $K$-vector. The charge density and the potential were Fourier expanded up to $G_{\max }=12 \mathrm{Ry}^{\frac{1}{2}}$. The crystal structures of rare gas solids $\mathrm{Kr}$ and $\mathrm{Ar}$ are shown in Fig. 1a, b. Special $k$-points were taken in the irreducible Brillouin zone (IBZ). For optical calculation, dens meshes are used to take account of the weak transitions.

We perform our study using the FP-LAPW method within density functional theory (DFT) [8, 16-19] as implemented in Wien2k package that has been proved to yield reliable results for electronic, optical, and structural properties of various solids [14, 20-25]. We use TB-mBJ approximation $[6,26]$ as the exchange potential plus LDA and GGA-PBE(96) exchange correlation potentials to do calculation. The self consistent calculations are considered to be converged when the difference in the total energy of the crystal is less than $0.0001 \mathrm{Ry}$.

\section{Results and discussion}

\subsection{Electronic properties}

In order to study the electronic properties, calculated band structure of rare gas solids Ar and $\mathrm{Kr}$ clusters are represented in Fig. 2a, b. One can
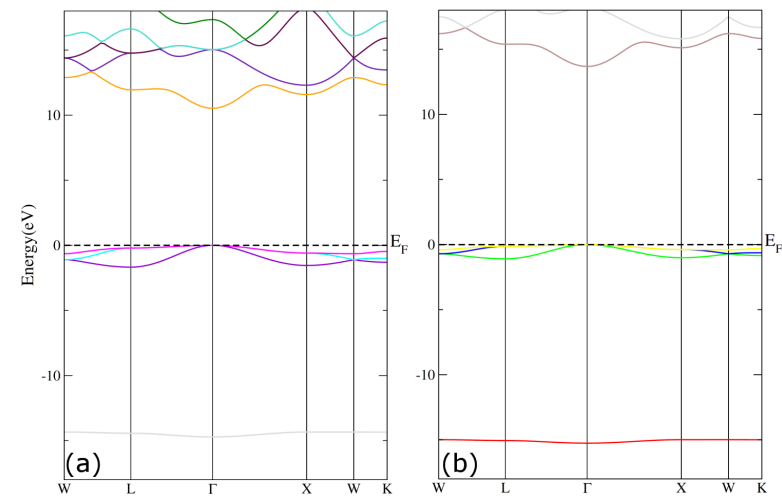

Fig. 2. Calculated band structures of rare gas solids (a) Kr and (b) Ar by using TB-mBJ approximation plus LDA exchange correlation potential.

TABLE I

The band gap energies for solid rare gas $\mathrm{Kr}$ and Ar rare gas solids obtained in the present work are compared with experimental and theoretical results. The approaches, i.e., TB-mBJ(LDA), TB$\mathrm{mBJ}(\mathrm{GGA}(96)), E_{g}^{\mathrm{avg}}$, considered in this work provide to the same final results.

\begin{tabular}{l|c|c}
\hline \hline & $\mathrm{Kr}$ & $\mathrm{Ar}$ \\
\hline this work & 10.523 & 13.673 \\
\hline \multirow{3}{*}{ experimental } & $11.59[3]$ at $30 \mathrm{~K}$ & $14.15[3]$ at $7 \mathrm{~K}$ \\
& $11.396[27]$ & $13.804[27]$ \\
& $11.6[6]$ & $14.20[6,29]$ \\
\hline \multirow{2}{*}{ theoretical } & $10.83[6]$ & $13.91[6]$ \\
& & $13.1[28]$
\end{tabular}

see there the insulator behavior of the two clusters. The maximum of the valence band and minimum of the conduction band are situated at the same high symmetry $\Gamma$ point, indicating that the two rare gas solids have a direct band gap.

The band gap of rare gas solids $\mathrm{Kr}$ and $\mathrm{Ar}$ calculated with TB-mBJ (LDA) and TB-mBJ (GGA(96)) are summarized in Table I, and compared with experimental and theoretical results. To obtain the average values in this work we applied the following formula:

$$
X_{\mathrm{avg}}=\frac{1}{2}\left(X_{\mathrm{TB}-\mathrm{mBJ}(\mathrm{LDA})}+X_{\mathrm{TB}-\mathrm{mBJ}(\mathrm{GGA}(96))}\right) .
$$

It can be seen from Table I that the values obtained within TB-mBJ(LDA) are in good agreement with previous theoretical results calculated within mBJ(LDA) [6]. These results are in good agreement with the available experimental and theoretical data $[3,6,27-29]$. The deviation percentage in obtained average energy gaps is about $1.01 \%$ and $3.48 \%$ from experimental values for rare gas solids $\mathrm{Kr}$ and Ar, respectively. Then, we can see that TB-mBJ (LDA and GGA$\mathrm{PBE}$ ) schemes give a good agreement with available experimental results. 

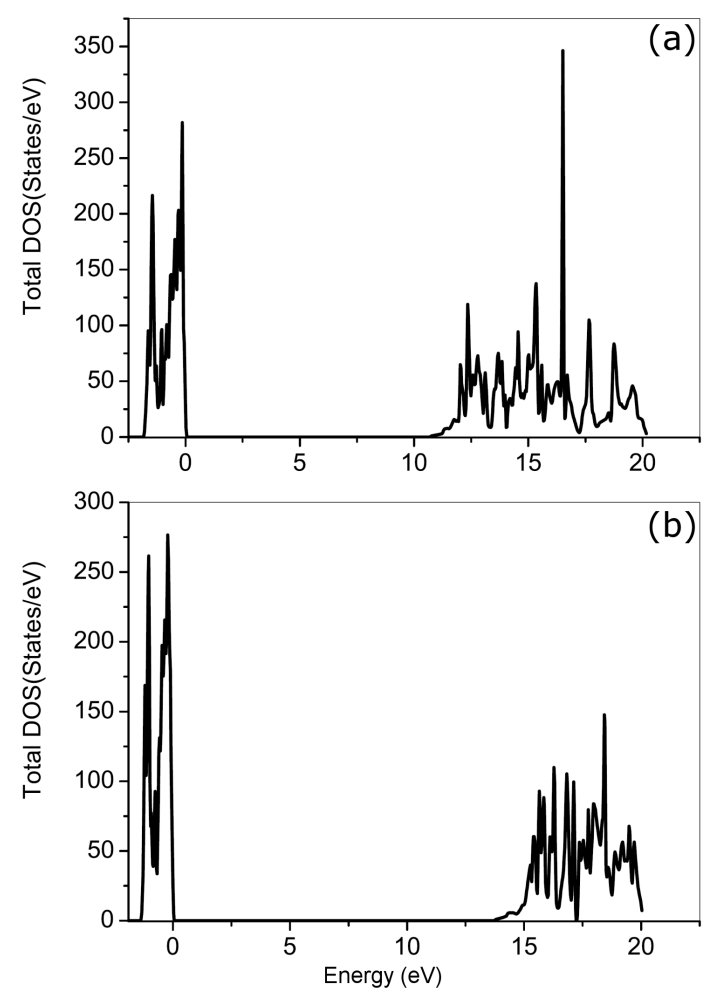

Fig. 3. Total density of states of (a) $\mathrm{Kr}$ and (b) Ar rare gas solids by using TB-mBJ approximation plus LDA exchange correlation potential.

Figure $3 \mathrm{a}, \mathrm{b}$ shows the total density of states of the rare gas solids $\mathrm{Kr}$ and $\mathrm{Ar}$, respectively within TB-mBJ (LDA) calculus. One can notice that the maximum of the valence band and the minimum of the conduction band are separated by the forbidden band.

The energy gaps are equal to $10.523 \mathrm{eV}$ and $13.673 \mathrm{eV}$ for $\mathrm{Kr}$ and $\mathrm{Ar}$ rare gas solids, respectively. The two rare gas solids have large band gap, and the calculated band gap energy within the two approximations describes correctly experimental results.

\subsection{Optical properties}

The optical properties of the studied rare gas solids (Ar and $\mathrm{Kr}$ ) are determined by frequency dependent of the dielectric function $\varepsilon(w)$ given by

$$
\varepsilon(w)=\varepsilon_{1}(w)+\mathrm{i} \varepsilon_{2}(w),
$$

where $\varepsilon_{1}(w)$ is the real part of the dielectric function which can be derived from the imaginary part $\varepsilon_{2}(w)$ by the Kramers-Kronig relations, $\varepsilon_{2}(w)$ can be obtained from the electronic structure calculation using the joint density of states and optical matrix elements. The real and imaginary parts of the dielectric function are given by [30-32]:

$$
\varepsilon_{1}(w)=1+\frac{2}{\pi} \int_{0}^{\infty} \frac{\varepsilon_{2}\left(w^{\prime}\right) w^{\prime} \mathrm{d} w^{\prime}}{w^{\prime 2}-w^{2}}
$$

$$
\begin{aligned}
& \varepsilon_{2}(w)=\frac{V e^{2}}{2 \pi m^{2} w^{2}} \int \mathrm{d}^{3} k \sum_{n n^{\prime}}\left|\left\langle k n|p| k n^{\prime}\right\rangle\right|^{2} \\
& \quad \times f(k n)\left(1-f\left(k n^{\prime}\right)\right) \partial\left(E_{k n}-E_{k n^{\prime}}-\hbar w\right),
\end{aligned}
$$

where $p$ is the momentum operator between states of $n$ and $n^{\prime}, e$ is the electron charge, $m$ is the mass of the electron, $w$ is the photon frequency, $V$ is the volume, $f(k n)$ is the Fermi distribution function and $|k n\rangle$ is the eigenfunction with eigenvalue $E_{k n}$. The refractive index $n(w)$ and the extinction coefficient $k(w)$ are given by $[33,34]$ :

$$
\begin{aligned}
& n(w)=\sqrt{\frac{\sqrt{\varepsilon_{1}^{2}(w)+\varepsilon_{2}^{2}(w)}+\varepsilon_{1}(w)}{2},} \\
& k(w)=\sqrt{\frac{\sqrt{\varepsilon_{1}^{2}(w)+\varepsilon_{2}^{2}(w)}-\varepsilon_{1}(w)}{2}} .
\end{aligned}
$$

The reflectivity $R(w)$, the absorption coefficient $\alpha(w)$ and the energy loss function $L(w)$ are defined by the following relations $[33,35]$ :

$$
\begin{aligned}
& R(w)=\frac{(n(w)-1)^{2}+k^{2}(w)}{(n(w)+1)^{2}+k^{2}(w)}, \\
& \alpha(w)=\frac{2 w k(w)}{c}=\frac{\varepsilon_{2}(w) w}{n(w) c}, \\
& L(w)=\frac{\varepsilon_{2}(w)}{\varepsilon_{1}^{2}(w)+\varepsilon_{2}^{2}(w)} .
\end{aligned}
$$

In the limit, at zero frequency, we get the following relation for the static refractive index:

$$
n(0)=\sqrt{\varepsilon_{1}(0)}
$$

The real and imaginary parts dependence of energy are shown in Fig. 4a, b for Kr and Fig. 4c, d for Ar rare gas solids respectively within TB-mBJ (LDA) and TB-mBJ (GGA(96)). From Fig. 4a, c, and at $0 \mathrm{eV}, \varepsilon_{1}(w)$ starts from 2.066 for $\mathrm{Kr}$ and from 1.627 for Ar within TB-mBJ(LDA) calculus. The static values of the real part of the dielectric function are given in Table II.
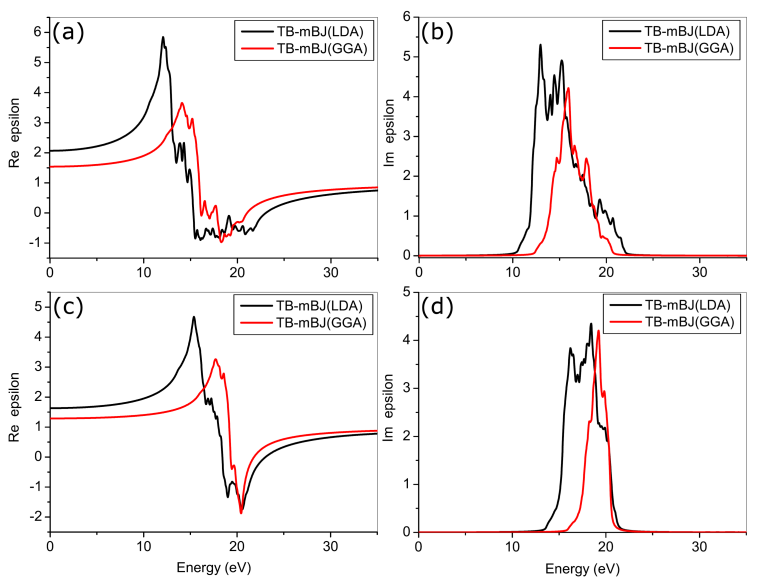

Fig. 4. Real (a) Kr (c) Ar and imaginary (b) Kr, (d) Ar parts of the dielectric function as a function of energy by using TB-mBJ approximation plus LDA and GGA(96) exchange correlation potentials. 
TABLE II

The calculated static dielectric constant $\varepsilon_{1}(0)$ and plasma frequency from the position of plasma peaks appearing in energy loss function $L(w)$.

\begin{tabular}{c|c|c|c}
\hline \hline \multicolumn{4}{c}{ Static dielectric constant } \\
\hline & TB-mBJ(LDA) & TB-mBJ(GGA $(96))$ & $\varepsilon_{1}^{\text {avg }}(0)$ \\
\hline $\mathrm{Kr}$ & 2.066 & 1.544 & 1.805 \\
$\mathrm{Ar}$ & 1.627 & 1.299 & 1.208 \\
\hline \multicolumn{4}{|c}{ Plasma frequency $w_{p}[\mathrm{eV}]$} \\
\hline & TB-mBJ(LDA) & TB-mBJ $(\mathrm{GGA}(96))$ & $w_{p}^{\text {avg }}$ \\
\hline $\mathrm{Kr}$ & 23.015 & 21.060 & 22.037 \\
$\mathrm{Ar}$ & 23.033 & 21.829 & 22.431
\end{tabular}

In Table II, we can see that the static values of the real part of the dielectric function have substantial differences at lower energy $0-7 \mathrm{eV}$ between TB-mBJ (LDA) and TB-mBJ (GGA(96)) for the two rare gas solids. The studied material have a large band gap, $\varepsilon_{1}(w)$ should decrease with increasing band gaps, then the low frequency optical dielectric constants are small.

The maximum of the peaks obtained within TB$\mathrm{mBJ}(\mathrm{LDA})$ are more important than those obtained within TB-mBJ(GGA(96)). The maximum values of the real part of the dielectric function within TB-mBJ (LDA) are small compared with those obtained within TB-mBJ(GGA(96)), for the two rare gas solids, corresponding to transitions from the valence to conduction bands. After this maximum, $\varepsilon_{1}(w)$ decreases and becomes negative within the two approximations for $\mathrm{Kr}$ and $\mathrm{Ar}$ rare gas solids, respectively, and the electromagnetic waves are reflected and there is no propagation in the medium $[21,36]$. From these results, we can see that there is a slightly difference between calculations within TB-mBJ (LDA) and TB-mBJ (GGA(96)). In fact, in the regions $0-10 \mathrm{eV}$ and $0-12 \mathrm{eV}$ also 23-35 eV for $\mathrm{Kr}$ and Ar rare gas solids within the two approximations, $\varepsilon_{2}(w)$ is zero and $\varepsilon_{1}(w)$ is positive, we can see that the extinction coefficient is zero and then we get the following relation for the refractive index:

$$
n(w)=\sqrt{\varepsilon_{1}(w)} .
$$

When the energy is smaller than $1 \mathrm{eV}$, the TB-mBJ (LDA) values of $\varepsilon_{1}(w)$ are slightly greater than those with TB-mBJ (GGA(96)).

The values of the maximum peaks of the imaginary part of the dielectric function, represented in Fig. 4b-d, for $\mathrm{Kr}$ and Ar rare gas solids within TBmBJ (LDA) are greater than those within TB-mBJ $($ GGA(96)). These maximum values of the peaks of $\varepsilon_{2}(w)$ are usually referred to the direct optical transitions between the top valence and the bottom conduction bands. At high energy the difference becomes small. After $21 \mathrm{eV} \varepsilon_{2}(w)$ becomes zero.

The reflectivity $R(w)$ and absorption coefficient $\alpha(w)$ as a function of energy are illustrated in Fig. $5 \mathrm{a}-\mathrm{d}$, the same small values were found in the
TABLE III

The calculated static refractive index $n(0)$ and the maximum values of reflectivity $R(w)$.

\begin{tabular}{c|c|c|c}
\hline \hline \multicolumn{4}{c}{ Refractive index } \\
\hline & TB-mBJ(LDA) & TB-mBJ(GGA $(96))$ & $n^{\text {avg }(0)}$ \\
\hline $\mathrm{Kr}$ & 1.436 & 1.239 & 1.337 \\
$\mathrm{Ar}$ & 1.280 & 1.136 & 1.208 \\
\hline \multicolumn{4}{c}{ Maximum values of $R(w)$} \\
\hline & TB-mBJ(LDA) & TB-mBJ $(\mathrm{GGA}(96))$ & $R^{\text {avg }(\mathrm{w})}$ \\
\hline $\mathrm{Kr}$ & 0.857 & 0.718 & 0.787 \\
$\mathrm{Ar}$ & 0.888 & 0.814 & 0.851
\end{tabular}
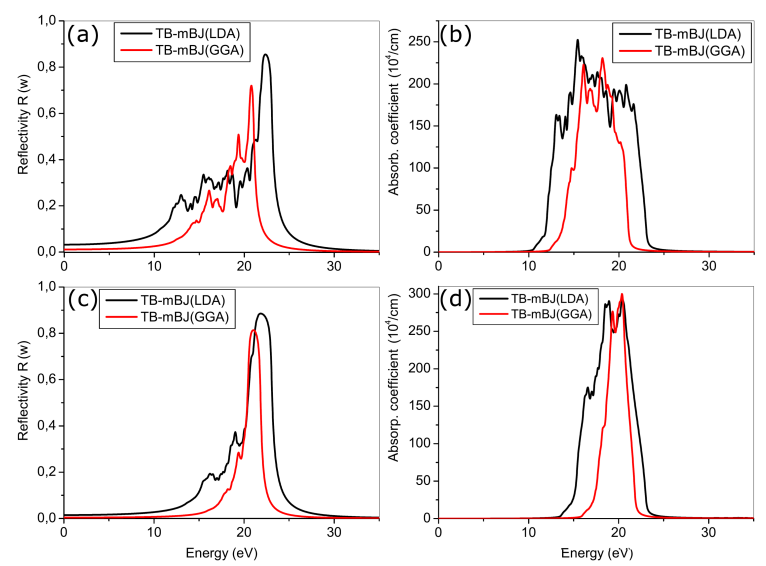

Fig. 5. Reflectivity (a) Kr, (c) Ar and absorption coefficient, (b) Kr, (d) Ar as a function of energy by using TB-mBJ(LDA and GGA(96)) approximations.

spectral band $0-7 \mathrm{eV}$, indicating that the two rare gas solids are transparent in infrared, visible and ultraviolet. The maximum values of $R(w)$ are summarized in Table III.

The energy variation of the absorption coefficient $\alpha(w)$ for both rare gas solids are shown in Fig. $5 \mathrm{c}, \mathrm{d}$. In the spectral band $0-7 \mathrm{eV}$, the absorption coefficient has insignificant values for both rare gas solids. The strongest peaks of $\alpha(w)$ occurs at $15.365 \mathrm{eV}$ and $20.389 \mathrm{eV}$ for rare gas solids $\mathrm{Kr}$ and Ar, respectively within TB-mBJ (LDA). However, the maximum values of the absorption coefficient are in the spectral bands $14-20 \mathrm{eV}$ and $19-20 \mathrm{eV}$, with the strongest peaks occurs at $18.178 \mathrm{eV}$ and $20.389 \mathrm{eV}$ within TB-mBJ (GGA(96)) for $\mathrm{Kr}$ and Ar, respectively. From these results, we can see that we have a small absorption in infrared, visible, and ultraviolet ranges $(0-7 \mathrm{eV})$, also we have found that the reflectivity coefficient $R(w)$ is small in this spectral region, within the two approximations. These results indicate that the two rare gas solids are transparent in the infrared, visible, and ultraviolet bands (0-7 eV), which suggests that these rare gas solids could be candidate for applications in optoelectronic devices. 

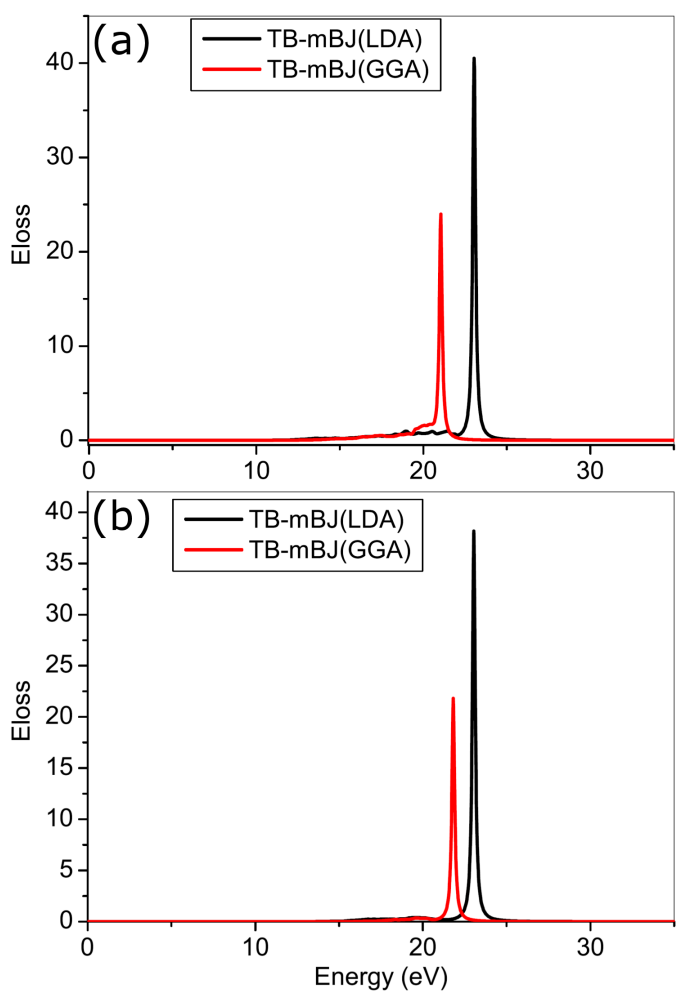

Fig. 6. Energy loss function as a function of energy by using TB-mBJ(LDA and GGA(96)) approximations for rare gas solids (a) Kr and (b) Ar.

The energy loss function $L(w)$, which describes the loss energy during the inelastic interaction of fast electrons with the medium, as a function of energy for rare gas solids $\mathrm{Kr}$ and $\mathrm{Ar}$ is shown in Fig. 6a, b. We have found the same behavior for the two studied rare gas solids within the two approximations. In the spectral region $0-20 \mathrm{eV}$ for $\mathrm{Kr}$ and Ar rare gas solids, $L(w)$ has very low values, but it increases rapidly and reaches its maximum, 40.452 and 37.889 within TB-mBJ (LDA), and 23.859 and 21.668 within TB-mBJ (GGA(96)) for $\mathrm{Kr}$ and Ar rare gas solids, respectively. The positions of these maximum values of the two peaks determine the plasma frequency of the material. The values of the plasma frequency for the two rare gas solids $\mathrm{Kr}$ and Ar within TB-mBJ (GGA(96)) and TB-mBJ (LDA) schemes and their average values are summarized in Table II.

The refractive index $n(w)$, which is an important factor in the optical applications for optoelectronic devices, and extinction coefficient $k(w)$ as a function of energy are illustrated in Fig. $7 \mathrm{a}-\mathrm{d}$. The refractive index represents the propagation behavior of light in materials. The static values of $n(w)$ at $0 \mathrm{eV}$ and their average values $n_{\text {avg }}$ for both rare gas solids and both approximations are given in Table III.

In Table III, we can see that the static values within TB-mBJ (LDA) are slightly greater than those within TB-mBJ (GGA(96)), because the refractive index is inversely proportional to the energy
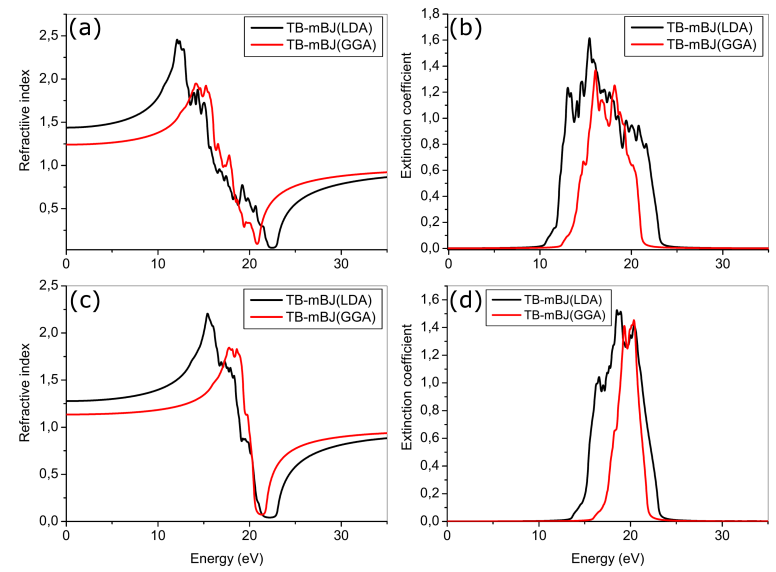

Fig. 7. Refractive index (a) Kr, (c) Ar and extinction coefficient (b) Kr, (d) Ar as a function of energy within TB-mBJ approximation plus LDA and GGA(96) exchange correlation potentials.

gap [37-39]. The maximum values of $n(w)$, which are related to the electron transitions from valence to conduction bands, within TB-mBJ (LDA), are greater than those within TB-mBJ (GGA(96)) for the two rare gas solids.

The maximum values of the extinction coefficient $k(w)$, represented in Fig. 7b-d, are 1.612 and 1.520, also 1.365 and 1.450 within TB-mBJ (LDA) and TB-mBJ (GA(96)), for the two rare gas solids $\mathrm{Kr}$ and Ar, respectively. At the interval $23-30 \mathrm{eV}$, $k(w)$ becomes very small for the two rare gas solids within the two approximations.

\section{Conclusion}

In this study, we have investigated the electronic and optical properties of rare gas solids $\mathrm{Kr}$ and $\mathrm{Ar}$ using the FP-LAPW method based on DFT within TB-mBJ (LDA and GGA(96)) exchange correlation potentials. The calculations provide a good description of the band structures and optical properties of the studied rare gas solids. The electronic properties are in good agreement with available theoretical and experimental results.

The use of TB-mBJ approach with LDA and GGA(96) exchange correlation potentials in the calculation of the electronic properties leads to a good results, the calculated band gap are much improved with TB-mBJ(LDA and GGA(96)) than other calculated band gap of the studied rare gas solids. We have found a direct band gap for the two rare gas solids located at the $\Gamma$ point. We have found that the optical properties depend strongly on the approximation used to calculate the band gap energy. The obtained results for the real and imaginary parts of the dielectric function and the other optical constants, within the two approximations, have the same behaviors for the two rare gas solids ( $\mathrm{Kr}$ and Ar) with some differences in their values. The real part of the dielectric function decreases with in- 
creasing band gap. It has been noticed that the two rare gas solids have a small absorption, a high transparency and small reflectivity in the infrared, visible, and ultraviolet ranges. For the present study, there is no available experimental or theoretical data for optical properties for the two rare gas solids to be compared with our results, it serves as a prediction for future studies.

\section{References}

[1] J.W.G. Tisch, N. Hay, K.J. Mendham et al., Nucl. Instrum. Methods Phys. Res. B 205, 310 (2003).

[2] M.H.R. Hutchinson, T. Ditmire, E. Springate, J.W.G. Tisch, Y.L. Shao, M.B. Mason, N. Hay, J.P. Marangos, Philos. Trans. R. Soc. Lond. A 356, 297 (1998).

[3] S. Bernstorff, V. Saile, Opt. Commun. 58, 181 (1986).

[4] S. Galamic-Mulaomerovic, C.H. Patterson, Phys. Rev. B 71, 195103 (2005).

[5] R.J. Magyar, A. Fleszar, E. Gross, Phys. Rev. B 69, 045111 (2004).

[6] F. Tran, P. Blaha, Phys. Rev. Lett. 102, 226401 (2009).

[7] A.D. Becke E.R. Johnson, J. Chem. Phys. 124, 221101 (2006).

[8] W. Kohn, L.J. Sham, Phys. Rev. 140, A1133 (1965).

[9] J.P. Perdew, Y. Wang, Phys. Rev. B 45, 13244 (1992).

[10] J.P. Perdew, K. Burke, M. Ernzerhof, Phys. Rev. Lett. 77, 3865 (1996).

[11] J.A. Camargo-Martinez, R. Baquero, Phys. Rev. B 86, 195106 (2012).

[12] D. Koller, F. Tran, P. Blaha, Phys. Rev. B 83, 195134 (2011).

[13] O.K. Anderson, Phys. Rev. B 12, 3060 (1975).

[14] K. Schwarz, P. Blaha, G.K.H. Madsen, Comput. Phys. Commun. 147, 71 (2002).

[15] P. Blaha, K. Schwarz, G.K.H. Madsen, D. Kvasnicka, J. Luitz, "WIEN2k, An augmented plane wave + local orbitals program for calculating crystal properties", Vienna University of Technology, Vienna 2014.

[16] S. Cottenier, "Density Functional Theory and the Family of (L) APW-Methods, a Step-By-Step Introduction", Center for Molecular Modeling and Department of Materials Science and Engineering, Ghent University, Belgium 2002-2013.

[17] R. Dreizler, E.K.U. Gross, Density Functional Theory, Springer, New York 1995.
[18] F. Kootstra, P.L. de Boeij, J.G. Snijders, J. Chem. Phys. 112, 6517 (2000).

[19] C. Li, R. Requist, E.K.U. Gross, J. Chem. Phys. 148, 084110 (2018).

[20] S.A. Khandy, I. Islam, Z.S. Ganai, D.C. Gupta, K.A. Parrey, J. Electron. Mater. 47, 436 (2018).

[21] R. Khemici, Z. Chouahda, L. Tairi, F. Semari, B. Amimoura, S. Benlameri, H. Meradji, S. Ghemid, Chin. J. Phys. 56, 1033 (2018).

[22] M. Ameri, S. Mesbah, Y. Al-Douri, B. Bouhafs, D. Varshney, I. Ameri, Acta Phys. Pol. A 125, 1110 (2014).

[23] S. Gagui, B. Zaidi, B. Hadjoudja, B. Chouial, H. Meradji, S. Ghemid, C. Shekhar, Computat. Condens. Matter 22, e00433 (2020).

[24] F. Zami, L. Djoudi, M. Merabet, S. Benalia, M. Boucharef, R. Belacel, D. Rached, Optik Int. J. Light Electron Opt. 180, 873 (2019).

[25] L. Djoudi, A. Lachebi, B. Merabet, H. Abid, Acta Phys. Pol. A 122, 748 (2012).

[26] D. Koller, F. Tran, P. Blaha, Phys. Rev. B 85, 155109 (2012).

[27] N.C. Bacalis, D.A. Papaconstantopoulos, W.E. Pickett, Phys. Rev. B 38, 6218 (1988).

[28] M. Grüning, A. Marini, A. Rubio, J. Chem. Phys. 124, 154108 (2006).

[29] P. Hohenberg, W. Kohn, Phys. Rev. B 136, 864 (1964).

[30] R. Dalven, Phys. Rev. B 8, 6033 (1973).

[31] Y. Shen, Z. Zhou, J. Appl. Phys. 103, 074113 (2008).

[32] C. Ambrosch-Draxl, J.O. Sofo, Comput. Phys. Commun. 175, 1 (2006).

[33] F. Wooten, Optical Properties of Solids, Academic Press, New York 1972.

[34] A. Delin, A.O. Eriksson, R. Ahuja, B. Johansson, M.S. Brooks, T. Gasche, S. Auluck, J.M. Wills, Phys. Rev. B 54, 1673 (1996).

[35] S. Saha, T.P. Sinha, A. Mookerjee, Phys. Rev. B 62, 8828 (2000).

[36] C. Kittel, Introduction to Solid State Physics, Wiley, New York 2013.

[37] T.S. Moss, Phys. Status Solidi B 131, 415 (1985).

[38] P.J.L. Herve, L.K.J. Vandamme, Infrared Phys. Technol. 35, 609 (1994).

[39] N.M. Ravindra, P. Ganapathy, J. Choi, Infrared Phys. Technol. 50, 21 (2007). 